\title{
Design and Simulation of the Control Algorithm for Vehicles of Geophysical Exploration
}

\author{
Jie Liu \& Yang Lv \& Zekun Liu \\ College of Instrumentation \& Electrical Engineering, Jilin University, Changchun, China \\ Zhiyan Dong* \\ College of Mechanical Science and Engineering, Jilin University, Changchun, China \\ *Corresponding author: Zhiyan Dong
}

ABSTRACT: In this paper, we select the quadrotor as the airbone geophysical exploration platform. By comparing the current quadrotor control algorithm, the classical closed-loop PID theory is selected. And then the platform flight control system is divided into three control loops, that is, position control loop, attitude control loop, and rotation speed control loop, which are respectively designed PID controller. The results that the controller simulated in the MATLAB / Simulink platform verify the effectiveness of the designed controller. KEYWORD: geophysical exploration; quadrotor; platform modeling; algorithm simulation

\section{INTRODUCTION}

Vehicles have become more and more widely used in the geophysical exploration work of harsh environment, and this technology has been a short board to our country (Amir M Y et al, 2008). Of the technology, the control algorithm is the most important one. For this reason, we took a deep study about the flight control algorithm in this article. Because of the big tail moment influenced, the unmanned helicopter is not suitable for low-flying, and with the reason of poor mobility, fixed wing UAV is largely influenced by the field limiting when taking-off and landing. So considering all above reasons, this paper chooses quadrotor as airbone geophysical exploration platform. Based on the classical PID control theory, this paper designs a control algorithm suitable for the system. The PID control algorithm has a completely theoretical system, (Mian A A et al, 2008) and the control structure is simple, good stability, and easy to adjust. Thanks to the strong antijamming and robustness in practical application, it has been widely used in the field of mechanical control.

\section{DESIGN OF CONTROL ALGORITHM}

\subsection{Analysis of control system}

The platform system is controlled by changing the four rotors speed to change the lift in four different directions, and then changing the motion platform so that the platform reaches the target position. In order to reduce the influence of the mutual coupling gen- erated by flight attitude (MD Schmidt, 2011), the whole platform flight control system is divided into three control loops (Do Nascimento D A et al, 2012) (Tezkan B. et al, 2011), namely rotation speed control loop, attitude control loop, and position control loop.

The rotation speed control loop is to control the four rotation speeds of the platform $\omega_{i}^{d}(i=1,2,3,4)$. The attitude control loop is to control the translational movement of the platform $\left(\begin{array}{llll}X & Y & Z\end{array}\right)$. The position control loop is to control the rotational displacement of the platform $\left(\begin{array}{lll}\phi & \theta & \psi\end{array}\right)$. The whole flying control system of the platform is showed in Fig.1.

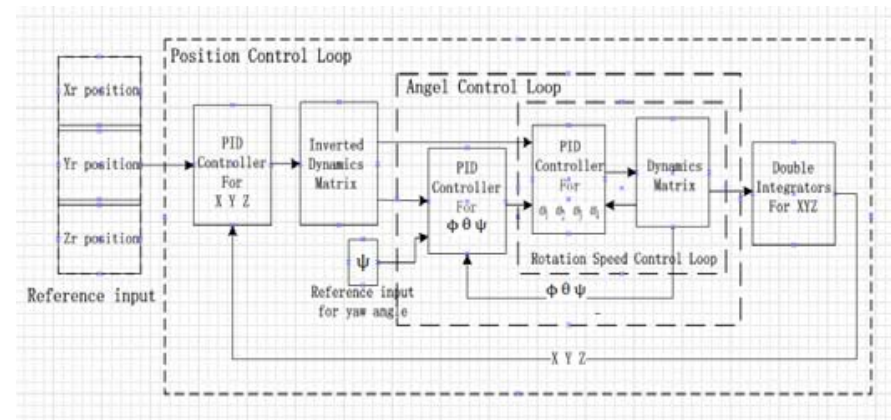

Fig.1 Flying control system of the platform

\subsection{Model of the platform}

In order to describe the kinematic relationship of quadrotor, firstly, we need to define two coordinate systems, respectively ground (inertial) coordinate 
system E-frame and body coordinate system Bframe, as shown in Fig.2.

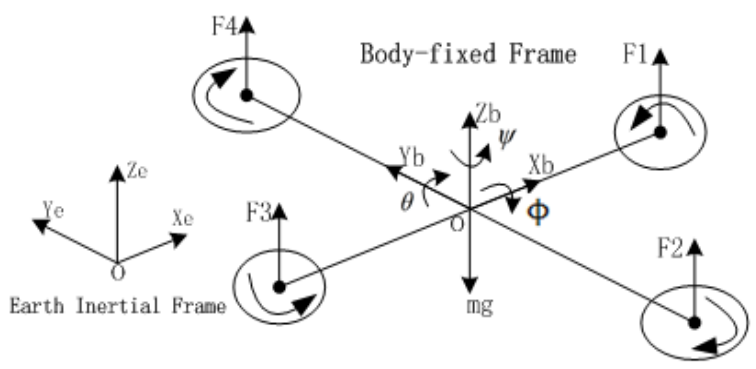

Fig.2 Coordinate systems of the platform

According to the definition of coordinate systems, whereby the introduction of six degrees of freedom of the aircraft, three position $\mathrm{X}_{\mathrm{e}}=[\mathrm{x} \mathrm{y} \mathrm{z}]^{\mathrm{T}}$ (represents the centroid of the aircraft with respect to the spatial position of the inertial coordinate system) and three angles $\eta_{\mathrm{e}}=\left[\begin{array}{lll}\varphi & \theta & \psi\end{array}\right]^{\mathrm{T}}$ (shows the relationship between the body coordinate system and the inertial coordinate system, usually Euler angles: $\varphi$ is the roll angle; $\theta$ is the pitch angle; $\psi$ is the yaw angle).

The rotational matrix $R_{B-E}$ from the body coordinate system B-frame to the inertial coordinate system E-frame can be expressed as follows:

$R_{B-E}=\left[\begin{array}{ccc}\mathrm{c} \theta \mathrm{c} \psi & \mathrm{s} \theta \mathrm{s} \phi \mathrm{c} \psi-\mathrm{c} \phi \mathrm{s} \psi & \mathrm{s} \theta \mathrm{c} \phi \mathrm{c} \psi+\mathrm{s} \phi \mathrm{s} \psi \\ \mathrm{c} \theta \mathrm{c} \psi & \mathrm{s} \theta \mathrm{s} \phi \mathrm{c} \psi+\mathrm{c} \phi \mathrm{s} \psi & \mathrm{s} \theta \mathrm{c} \phi \mathrm{s} \psi-\mathrm{s} \phi \mathrm{c} \psi \\ -\mathrm{s} \theta & \mathrm{s} \phi \mathrm{c} \theta & \mathrm{c} \phi \mathrm{c} \theta\end{array}\right]$

Where we use $s \phi, s \theta, s \psi$ for $\sin \phi, \sin \theta, \sin \psi$ and $c \phi$, $c_{\theta}, c_{\psi}$ for $\cos \phi, \cos \theta, \cos \psi$.

The dynamical model of the platform is based on the Newton-Euler equations as follows:

$$
\left\{\begin{array}{l}
\sum F^{b}=m \dot{V}^{b}+W^{b} \times\left(m V^{b}\right) \\
\sum M^{b}=I \dot{W}^{b}-W^{b} \times\left(I W^{b}\right)
\end{array}\right.
$$

Where $m$ denotes the mass of the platform; $\sum F^{b}$ demotes the total body force; $\dot{V}^{b}$ denotes the body's linear acceleration; $V^{b}$ demotes the body line speed, $V^{b}=\left[\begin{array}{lll}u & v & w\end{array}\right]^{T} ; \sum M^{b}$ demotes the total body moment; $\dot{W}^{b}$ demotes the body angular acceleration; $W^{b}$ demotes the body angular velocity, $W^{b}=\left[\begin{array}{ccc}p & q & r\end{array}\right]^{T} ; I$ demotes the body's inertia moment matrix, $I=\left[\begin{array}{ccc}I_{X X} & 0 & 0 \\ 0 & I_{Y Y} & 0 \\ 0 & 0 & I_{Z Z}\end{array}\right]$.

For the model set up in the indoor flight state, ignore the influence of air resistance. The force of the platform system analysis shows that the main force of the system includes lift force generated by the propellors rotation and gravity force . The lift force is $F_{T}^{b}=\left[\begin{array}{lll}0 & 0 & \sum_{i=1}^{4} F_{i}\end{array}\right]^{T}$

Where $F_{i}(\mathrm{i}=1,2,3,4)$ for thrust generated by propeller, $F_{i}=b \omega_{i}^{2}$ ( $b$ for propeller lift factor, $\omega$ for propeller rotation speed).

$F_{G}^{e}=\left[\begin{array}{lll}0 & 0 & -m g\end{array}\right]^{T}$

Transform the thrust $F_{T}^{b}$ and gravity $F_{G}^{e}$ by the rotation matrix, it follows that

$$
\begin{aligned}
& F_{T}^{e}=R_{b-e} F_{T}^{b}=R_{b-e}\left[\begin{array}{lll}
0 & 0 & \sum_{i=1}^{4} F_{i}
\end{array}\right]^{T} \\
& =\sum_{i=1}^{4} F_{i}\left[\begin{array}{c}
\sin \phi \sin \psi+\cos \phi \sin \theta \cos \psi \\
-\sin \phi \cos \psi+\cos \phi \sin \theta \sin \psi \\
\cos \phi \cos \theta
\end{array}\right] \\
& F_{G}^{b}=R_{e-b} F_{G}^{e}=R_{e-b}\left[\begin{array}{lll}
0 & 0 & -m g
\end{array}\right]^{T} \\
& =m g\left[\begin{array}{c}
\sin \theta \\
-\cos \theta \sin \phi \\
-\cos \theta \cos \phi
\end{array}\right]
\end{aligned}
$$

Where $R_{e-b}=R_{b-e}^{T}$.

Carrying formula (3) and formula (6) into the Newtonian equations of the formula (2), it follows that

$$
\left[\begin{array}{c}
\dot{u} \\
\dot{v} \\
\dot{w}
\end{array}\right]=\left[\begin{array}{c}
\mathrm{g} \sin \theta-q w+v r \\
-g \cos \theta \sin \phi-w r+p w \\
-g \cos \theta \cos \phi+\frac{1}{m} \sum_{i=1}^{4} F_{i}-p w+q u
\end{array}\right]
$$

According to Newton's second law $\sum F^{e}=m \frac{d V^{e}}{d t}$, rewrite formula (4) and formula (5), it follows that

$$
\left[\begin{array}{c}
\ddot{X} \\
\ddot{Y} \\
\ddot{Z}
\end{array}\right]=\left[\begin{array}{c}
\frac{1}{m} \sum_{i=1}^{4} F_{i}(\sin \phi \sin \psi+\cos \phi \sin \theta \cos \psi) \\
\frac{1}{m} \sum_{i=1}^{4} F_{i}(-\sin \phi \cos \psi+\cos \phi \sin \theta \sin \psi) \\
\frac{1}{m} \sum_{i=1}^{4} F_{i} \cos \phi \cos \theta-g
\end{array}\right]
$$

Where $V^{e}$ is for the body line speed in E-frame, $V^{e}=\left[\begin{array}{ccc}\dot{X} & \dot{Y} & \dot{Z}\end{array}\right]^{T}$.

The main sources of the outside moment of the platform come from drag torque, aerodynamic torque, and gyroscopic moment. Because of the symmetry axis of the body of the platform, the drag on the body does not produce any torque. Based on 
the special flight status of the platform, low-attitude and low-speed, the gyroscopic effect of the body is not obvious, so here we ignore the impact of gyroscopic effect. As a result, the moment of the platform is mainly due to the aerodynamic torque generated by the propeller rotation. The aerodynamic torque ${ }^{M_{T}}$ can be expressed as

$$
M_{T}^{b}=\left[\begin{array}{c}
l\left(F_{2}-F_{4}\right) \\
l\left(F_{1}-F_{3}\right) \\
M_{1}-M_{2}+M_{3}-M_{4}
\end{array}\right]
$$

Where $M_{i}=d \omega_{i}^{2}(i=1,2,3,4)$ for the propeller resistance moment, $d$ for the propeller resistance factor.

Rewrite the equation (9) with (2), it follows that

$$
\left[\begin{array}{c}
\dot{p} \\
\dot{q} \\
\dot{r}
\end{array}\right]=\left[\begin{array}{c}
\frac{l\left(F_{2}-F_{4}\right)}{I_{X X}}-\frac{q r\left(I_{Z Z}-I_{Y Y}\right)}{I_{X X}} \\
\frac{l\left(F_{1}-F_{3}\right)}{I_{Y Y}}-\frac{p r\left(I_{X X}-I_{Z Z}\right)}{I_{Y Y}} \\
\frac{M_{1}-M_{2}+M_{3}-M_{4}}{I_{Z Z}}-\frac{p q\left(I_{Y Y}-I_{X X}\right)}{I_{Z Z}}
\end{array}\right]
$$

According to the rotation theory, there is

$$
\left[\begin{array}{lll}
\dot{\phi} & \dot{\theta} & \dot{\psi}
\end{array}\right]^{T}=T_{b-e}\left[\begin{array}{lll}
p & q & r
\end{array}\right]^{T}
$$

Where $T_{b-e}$ is for the transition matrix,

$$
T_{b-e}=\left[\begin{array}{ccc}
1 & \tan \theta \sin \phi & \tan \theta \cos \phi \\
0 & \cos \phi & -\sin \phi \\
0 & \sin \phi / \cos \theta & \cos \phi / \cos \theta
\end{array}\right] .
$$

The Kinetic model equation of the platform can be expressed as follows:

$$
\begin{aligned}
& \left\{\begin{array}{l}
\ddot{X}=\frac{U_{1}}{m}(\sin \phi \sin \psi+\cos \phi \sin \theta \cos \psi) \\
\ddot{Y}=\frac{U_{1}}{m}(-\sin \phi \cos \psi+\cos \phi \sin \theta \sin \psi) \\
\ddot{Z}=\frac{U_{1}}{m} \cos \phi \cos \theta-g \\
\dot{p}=\frac{U_{2}}{I_{X X}}-\frac{q r\left(I_{Z Z}-I_{Y Y}\right)}{I_{X X}} \\
\dot{q}=\frac{U_{3}}{I_{Y Y}}-\frac{p r\left(I_{X X}-I_{Z Z}\right)}{I_{Y Y}} \\
\dot{r}=\frac{d U_{4}}{I_{Z Z}}-\frac{p q\left(I_{Y Y}-I_{X X}\right)}{I_{Z Z}} \\
U_{1}=\sum_{i=1}^{4} F_{i}=b\left(\omega_{1}^{2}+\omega_{2}^{2}+\omega_{3}^{2}+\omega_{4}^{2}\right) \\
U_{2}=l\left(F_{2}-F_{4}\right)=b l\left(\omega_{2}^{2}-\omega_{4}^{2}\right) \\
U_{3}=l\left(F_{1}-F_{3}\right)=b l\left(\omega_{1}^{2}-\omega_{2}^{2}\right) \\
U_{4}=M_{1}-M_{2}+M_{3}-M_{4}=d\left(\omega_{1}^{2}-\omega_{2}^{2}+\omega_{3}^{2}-\omega_{4}^{2}\right)
\end{array}\right.
\end{aligned}
$$

Wherein, to simplify the model description, definition four variables about the rotors $\operatorname{speed} U_{1}, U_{2}$, $U_{3}, U_{4}$ for the model input.

Considering the flight status of the platform, mostly in the low attitude, low speed or hover state, small angle change, so the change rate of the angle can be approximately zero and formula (10) can be seen as a unit matrix. And then the kinetic model of the platform system, formula (12) can be re-defined as:

$$
\left\{\begin{array}{l}
\ddot{X}=\frac{U_{1}}{m}(\sin \phi \sin \psi+\cos \phi \sin \theta \cos \psi) \\
\ddot{Y}=\frac{U_{1}}{m}(-\sin \phi \cos \psi+\cos \phi \sin \theta \sin \psi) \\
\ddot{Z}=\frac{U_{1}}{m} \cos \phi \cos \theta-g \\
\ddot{\phi}=\frac{U_{2}}{I_{X X}} \\
\ddot{\theta}=\frac{U_{3}}{I_{Y Y}} \\
\ddot{\psi}=\frac{d U_{4}}{I_{Z Z}}
\end{array}\right.
$$

\subsection{Design of control algorithm}

\section{1) Position control loop}

The position PID controller module adopts the PID control algorithm for the three input desired displacement $\left._{\left(X^{d}\right.} Y^{d} Z^{d}\right)$, and the specific form is as follows:

$$
\left\{\begin{array}{l}
\ddot{X^{d}}=k_{P X}\left(X^{d}-X\right)+k_{I X} \int\left(X^{d}-X\right) d t+k_{D X}\left(\dot{X^{d}}-\dot{X}\right) \\
\ddot{Y^{d}}=k_{P Y}\left(Y^{d}-Y\right)+k_{I Y} \int\left(Y^{d}-Y\right) d t+k_{D Y}\left(Y^{d}-\dot{Y}\right) \\
\ddot{Z^{d}}=k_{P Z}\left(Z^{d}-Z\right)+k_{I Z} \int\left(Z^{d}-Z\right) d t+k_{D Z}\left(\dot{Z^{d}}-\dot{Z}\right)
\end{array}\right.
$$

Where $k_{P X} \quad k_{P Y} \quad k_{P Z}$ denote the proportional coefficient, $k_{I X} \quad k_{I Y} \quad k_{I Z}$ denote the integral coefficient, $k_{D X} k_{D Y} \quad k_{D Z}$ denote the differential coefficient.

In the acceleration inverse solution module, it takes the output of position PID controller module $\left(\begin{array}{ccc}\ddot{X}^{d} & \ddot{Y}^{d} & \ddot{Z^{d}}\end{array}\right)$ as input, according to the formula (14) the high acceleration equation, $U_{1}$ can be calculated as follows:

$$
U_{1}=\frac{m}{\cos \phi \cos \theta}\left(\ddot{Z^{d}}-g\right)
$$

According to the formula (14) in the horizontal acceleration equation, $\phi^{d}$ can be calculate as follows:

$\phi^{d}=\arcsin \left[\frac{m}{U_{1}}\left(\ddot{X^{d}} \cos \psi^{d}-\ddot{Y^{d}} \sin \psi^{d}\right)\right]$

Where $\psi^{d}$ donates the reference value for a given desired yaw angle.

Take the first equation in formula (14) with (17), $\theta^{d}$ can be calculate as follows: 
$\theta^{d}=\arcsin \left(\frac{\left.\frac{m}{U_{1}} \ddot{X}^{d}-\sin \phi^{d} \sin \psi^{d}\right)}{\cos \phi^{d} \cos \psi^{d}}\right)$

2) Attitude control loop

As the core of the control system of the platform, the attitude control loop ensures the position control loop stability, and determines the platform flight status. Its control result is directly affected the quality of flight platform.

The attitude PID controller adopts the PID control algorithm for the three input desired angular displacement ( $\phi^{d} \quad \theta^{d} \quad \psi^{d}$ ), and the specific form is as follows:

$\left\{\begin{array}{l}U_{2}=k_{P \phi}\left(\phi^{d}-X\right)+k_{I \phi} \int\left(\phi^{d}-\phi\right) d t+k_{D \phi}\left(\dot{\phi^{d}}-\dot{\phi}\right) \\ U_{3}=k_{P \theta}\left(\theta^{d}-\theta\right)+k_{I \theta} \int\left(\theta^{d}-\theta\right) d t+k_{D \theta}\left(\dot{\theta^{d}}-\dot{\theta}\right) \\ U_{4}=k_{P \psi}\left(\psi^{d}-\psi\right)+k_{I \psi} \int\left(\psi^{d}-\psi\right) d t+k_{D \psi}\left(\dot{\psi^{d}}-\dot{\psi}\right)\end{array}\right.$

Where $k_{P \phi} \quad k_{P \theta} \quad k_{P \psi}$ denote the proportional coefficient, $k_{I \phi} \quad k_{I \theta} \quad k_{I \psi}$ denote the integral coefficient, $k_{D \phi} \quad k_{D \theta} \quad k_{D \psi}$ denote the differential coefficient.

In the speed of inverse solution module, it takes $U_{1}, U_{2}, U_{3}, U_{4}$ as input, according to formula (13), it follows that:

$$
\left\{\begin{array}{l}
\omega_{1}^{d_{1}}=\left(\frac{1}{4 b} U_{1}+\frac{1}{2 b l} U_{3}+\frac{1}{4 d} U_{4}\right)^{\frac{1}{2}} \\
\omega^{d}{ }_{2}=\left(\frac{1}{4 b} U_{1}+\frac{1}{2 b l} U_{2}-\frac{1}{4 d} U_{4}\right)^{\frac{1}{2}} \\
\omega^{d}{ }_{3}=\left(\frac{1}{4 b} U_{1}-\frac{1}{2 b l} U_{3}+\frac{1}{4 d} U_{4}\right)^{\frac{1}{2}} \\
\omega^{d}{ }_{4}=\left(\frac{1}{4 b} U_{1}-\frac{1}{2 b l} U_{2}-\frac{1}{4 d} U_{4}\right)^{\frac{1}{2}}
\end{array}\right.
$$

\section{3) Rotation speed control loop}

By changing the rate of rotation of the rotor, the platform can achieve various flights. In order to take a flexible response, the rotation speed control loop of the platform must have a quick adjusting ability.

Due to the short response time of the motor, the response time is much less than the movement cycle of the system platform, so instead of using the gain factor of the transfer function of the motor. P control algorithm is taken in the speed controller module, the specific form of control of the desired angular velocity $\omega_{i}^{d}$ is as follows:

$\omega_{i}=k_{P \omega i} \omega_{i}{ }^{d}$

Where $k_{P \omega i}$ denotes the proportional coefficient.

\section{SIMULATION OF CONTROL ALGORITHM}

We choose the MATLAB/SIMULINK software as the flight control algorithm of the airbone geophysical exploration platform simulation platform. Based on the simplified model of the platform and the designed closed-loop PID controllers, simuliate the platform control system on SIMULINK platform, the platform simulation module shown in Fig.3.

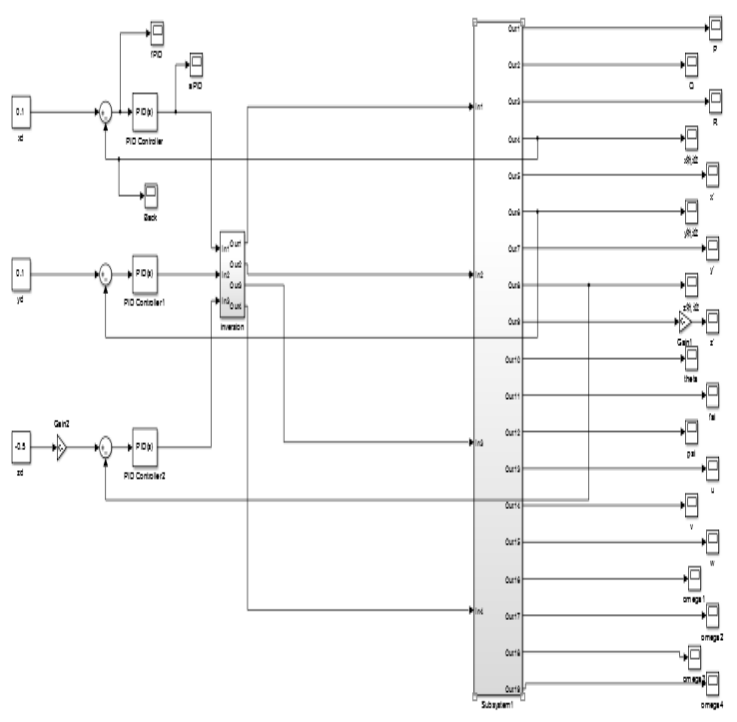

Fig.3 Simulation Module of the Platform System

Tab.1 Parameters Table of the Platform

\begin{tabular}{|l|ll|l|l|}
\hline$m$ & 0.725 & $\mathrm{~kg}$ & $J_{X}$ & $0.0196 \mathrm{~kg} / \mathrm{s}^{2}$ \\
\hline$l$ & 0.25 & $\mathrm{~m}$ & $J_{Y}$ & $0.0196 \mathrm{~kg} / \mathrm{s}^{2}$ \\
\hline $\mathrm{g}$ & 9.81 & $\mathrm{~m} / \mathrm{s}^{2}$ & $J_{Z}$ & $0.03938 \mathrm{~kg} / \mathrm{s}^{2}$ \\
\hline $\mathrm{b}$ & \multicolumn{2}{|l|}{$3.31 \times 10^{-5} \mathrm{~N}^{*} \mathrm{~s}^{2}$} & $\mathrm{~d}$ & $7.5 \times 10^{-7} \mathrm{~N}^{*} \mathrm{~s}^{2}$ \\
\hline
\end{tabular}

Before simulation, firstly, we must set initial state and desired destination path of the platform. The initial position is $\left(\begin{array}{lll}0 & 0 & 0\end{array}\right)$; the initial attitude angle is ( $\left.\begin{array}{lll}0.5 & 0.5 & 0.5\end{array}\right)$; the desired target position is $\left(\begin{array}{lll}1 & 1 & 1\end{array}\right)$; the desired yaw angle is $0 \mathrm{rad}$. The constant parameters using in simulation shown in Tab.1.

After several simulation and debugging tests, the coefficients of the PID controllers were determined, the specific values shown in Tab.2.

Tab.2 Parameters Table of the PID Controllers

\begin{tabular}{|l|l|l|l|l|l|}
\hline$k_{P X}$ & 2 & $k_{I X}$ & 0.8 & $k_{D X}$ & 2.2 \\
\hline$k_{P Y}$ & 2 & $k_{I Y}$ & 0.8 & $k_{D Y}$ & 2.2 \\
\hline$k_{P Z}$ & 1.5 & $k_{I Z}$ & 0.005 & $k_{D Z}$ & 3 \\
\hline$k_{P \phi}$ & 5 & $k_{I \phi}$ & 0.1 & $k_{D \phi}$ & 1.1 \\
\hline$k_{P \theta}$ & 5 & $k_{I \theta}$ & 0.1 & $k_{D \theta}$ & 1.1 \\
\hline$k_{P \psi}$ & 1.5 & $k_{I \psi}$ & 0.9 & $k_{D \psi}$ & 0.4 \\
\hline
\end{tabular}


The attitude response curve of the platform controller is shown in Fig.4.
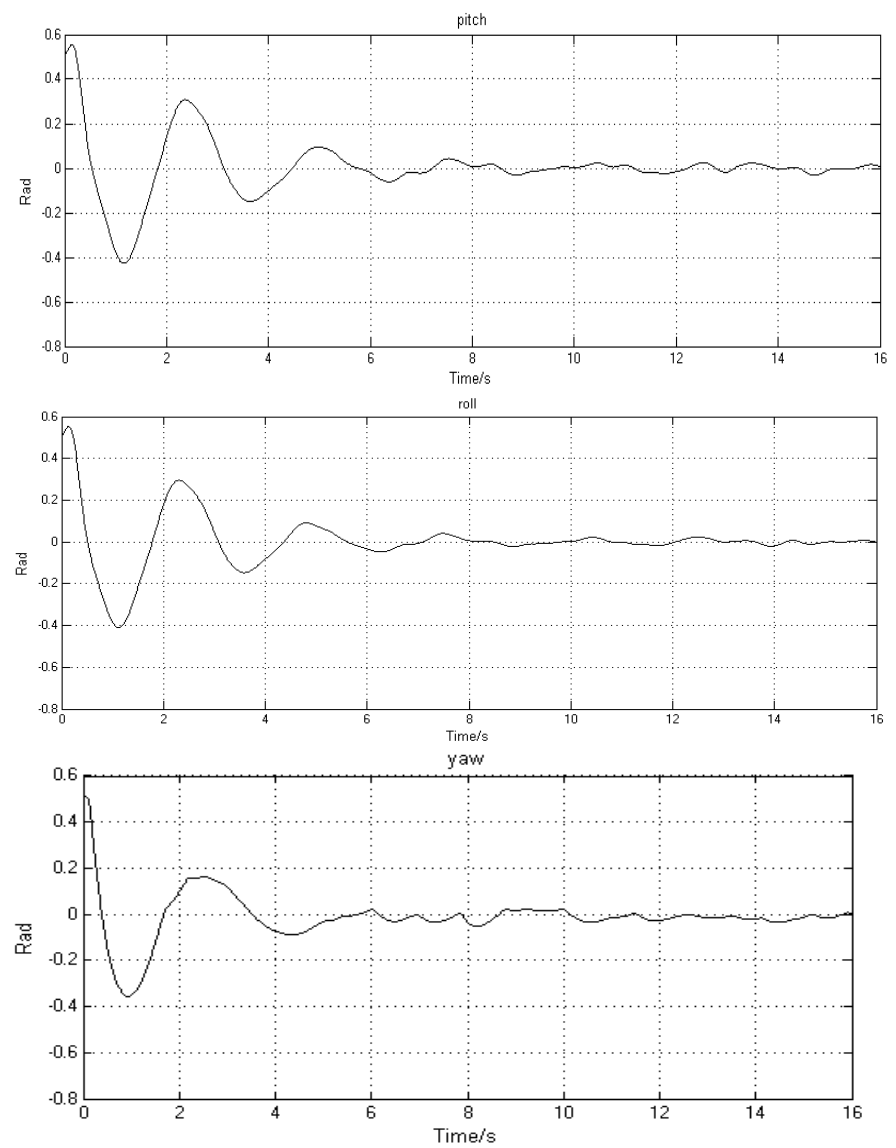

Fig.4 Attitude Response Curve of PID Controller

Figure 4 shows the attitude simulation results. It is shown that the design of attitude control loop has better control effect. Roll, pitch, yaw three control channels can quickly reach a steady state, and can be maintained in a stable state. Time response of the control loop is faster, better stability, and to meet the system's basic design requirements. The controller works well.

The position response curve of the platform controller is shown in Fig.4.

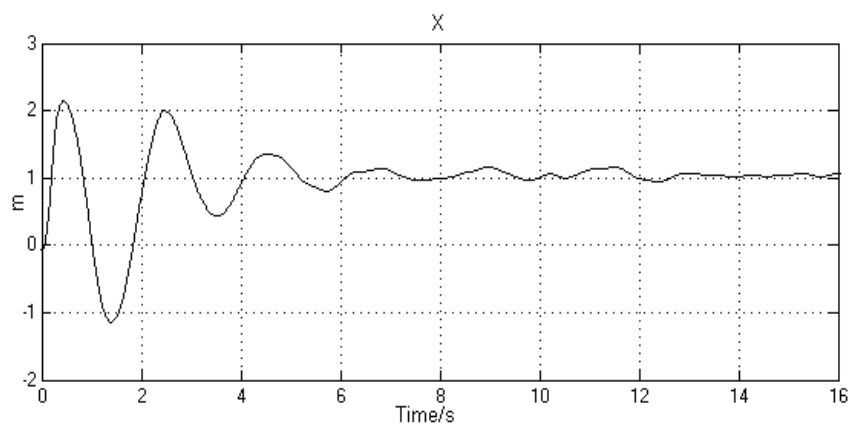

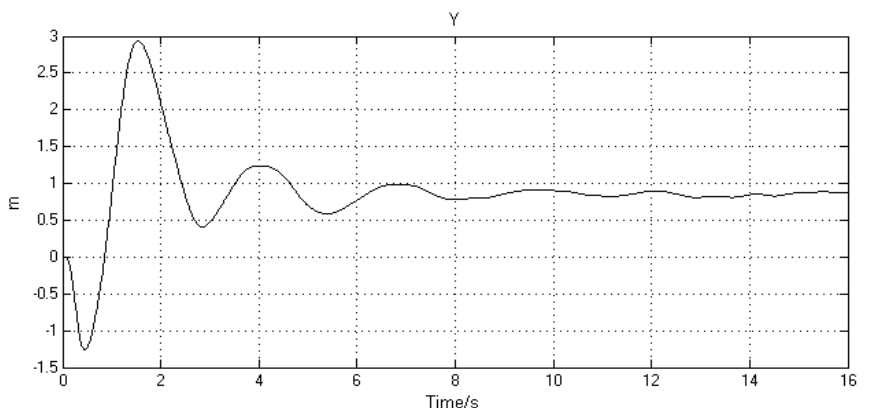

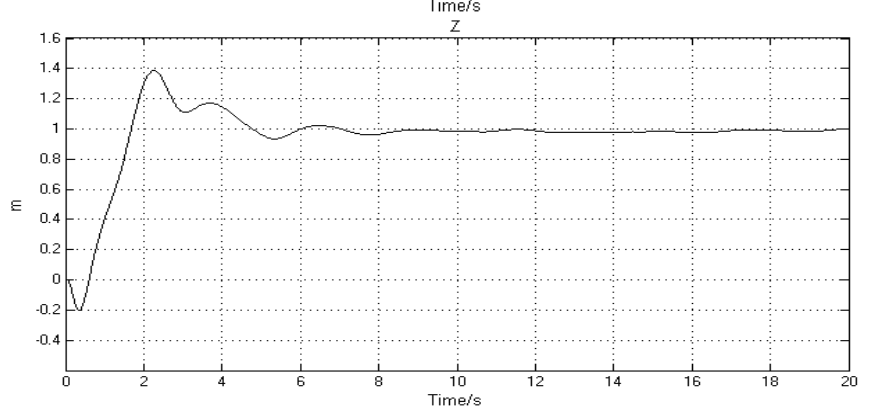

Fig.5 Position Response Curve of PID Controller

Fig. 5 shows the position simulation results. It is shown that the design of the position control loop has better automatic control ability. $\mathrm{X}, \mathrm{Y}, \mathrm{Z}$ response curve can quickly reach the desired target position, and to maintain a steady state. When in $6 \mathrm{~s}$ $\mathrm{Z}$ position of the state can achieve the desired target height, so the quick response feather of the position control loop meet the design requirements. The controller works well.

\section{CONCLUSION}

According to the using background of the airbone geophysical exploration platform, we design the flight controller of the platform. Firstly, the entire platform flight control system is divided into three control loops: position control loop, attitude control loop and speed control loop, and based on the classical closed-loop PID control theory do the flying controller design. Then the MATLAB/SIMULINK simulation platform is used to simulate the platform control system, According to the simulation results, the airbone geophysical exploration platform system can stably fulfill its mission under the designed PID controller.

\section{REFERENCES}

Amir M Y, Abbass V. Modeling of Quadrotor Helicopter Dynamics[C]// Smart Manufacturing Application, 2008. ICSMA 2008. International Conference on. 2008:100-105.

Do Nascimento D A, Rufino C Z, Oliveira S C, et al. Modeling and Design of an Autonomous Unmanned Aerial Vehicle Quadrotor Prototype for Applications in Swarm of Robots[C]// Robotics Symposium and Latin American Robotics Symposium (SBR-LARS), 2012 Brazilian. IEEE, 2012:325328. 
MD Schmidt. Simulation and Control of a Quadrotor Unmanned Aerial Vehivle[D]. University of Kentucky, 2011.

Mian A A, Daobo W. Nonlinear Flight Control Strategy for an Underactuated Quadrotor Aerial Robot[C]// Networking, Sensing and Control, 2008. ICNSC 2008. IEEE International Conference on. IEEE, 2008:938-942.

Tezkan B., Stoll J.B., Bergers R., and Grobbach H.. Unmanned aircraft system proves itself as a geophysical measuring platform for aeromagnetic surveys [J]. First Break, 2011, 29:103-105. 